\title{
СТУПЕНЧАТО-АФФИННЫЕ ФУНКЦИИ, ПОЛУПРОСТРАНСТВА И ОТДЕЛИМОСТЬ ВЫПУКЛЫХ МНОЖЕСТВ С ПРИЛОЖЕНИЯМИ К ВЫПУКЛЫМ ЗАДАЧАМ ОПТИМИЗАЦИИ ${ }^{1}$
}

\section{В. В. Гороховик}

\begin{abstract}
В статье приводится определение ступенчато-аффинных функций, определенных на вещественном векторном пространстве, и устанавливается их двойственность полупространствам - выпуклым множествам, дополнения которых также выпуклы. С использованием этой двойственности доказывается, что два выпуклых подмножества вещественного векторного пространства не пересекаются тогда и только тогда, когда они отделимы некоторой ступенчато-аффинной функцией. Фактически данный критерий непересекаемости выпуклых множеств является аналитическим вариантом критерия Какутани - Тьюки об отделимости непересекающихся выпуклых множеств полупространствами. В качестве приложений получены критерий минимальности решений для выпуклых задач векторной оптимизации, рассматриваемых в вещественных векторных пространствах без топологии, и критерий оптимальности допустимых точек в классических задачах выпуклого программирования, не удовлетворяющих условию регулярности Слейтера.

Ключевые слова: ступенчато-аффинные функции, полупространства, отделимость выпуклых множеств, выпуклые задачи векторной оптимизации, выпуклое программирование.

V. V. Gorokhovik. Step-affine functions, half-spaces, and separation of convex sets with applications to convex optimization problems.

We present the definition of step-affine functions defined on a real vector space and establish the duality between step-affine functions and half-spaces, i.e., convex sets whose complements are convex as well. Using this duality, we prove that two convex sets are disjoint if and only if they are separated by some step-affine function. This criterion is actually the analytic version of the Kakutani-Tukey criterion of the separation of disjoint convex sets by half-spaces. As applications of these results, we derive a minimality criterion for solutions of convex vector optimization problems considered in real vector spaces without topology and an optimality criterion for admissible points in classical convex programming problems not satisfying the Slater regularity condition.
\end{abstract}

Keywords: step-affine functions, half-spaces, separation of convex sets, convex vector optimization problems, convex programming.

MSC: 52A05, 52A41, 49K27, 90C25

DOI: $10.21538 / 0134-4889-2020-26-1-51-70$

\section{Введение}

Классические теоремы об отделимости выпуклых множеств гиперплоскостями играют ключевую роль не только в выпуклом анализе, но и в других разделах математики и ее многочисленных приложениях, в частности, в теории оптимизации. Геометрически отделимость двух подмножеств векторного пространства некоторой гиперплоскостью означает, что эти подмножества принадлежат различным алгебраически замкнутым полупространствам, порождаемым этой гиперплоскостью. Вследствие двойственности, существующей между гиперплоскостями и аффинными функциями, свойство отделимости может быть записано в аналитическом виде. В конечномерных векторных пространствах для любых двух непересекающихся выпуклых множеств существует отделяющая их гиперплоскость. В то же время в любом бесконечномерном векторном пространстве существуют непересекающиеся выпуклые множества,

\footnotetext{
${ }^{1}$ Работа выполнена в рамках Государственной программы научных исследований Республики Беларусь на 2016- 2020 годы “Конвергенция-2020" (проект 1.4.01).
} 
которые нельзя отделить никакой гиперплоскостью (см., например, [1, с.131]). По этой причине в целом ряде задач анализа применение классических теорем об отделимости либо оказывается вовсе невозможным, либо обеспечивается некоторыми дополнительными предположениями относительно данных задачи. Хорошо известно, что достаточным условием, обеспечивающим существование отделяющей гиперплоскости для двух непересекающихся выпуклых подмножеств бесконечномерного векторного пространства, является требование, чтобы хотя бы одно из них имело непустую алгебраическую внутренность.

Другой особенностью классических теорем об отделимости является то, что они, вообще говоря, необратимы: из отделимости множеств некоторой гиперплоскостью не следует то, что эти множества не пересекаются. Для того чтобы обеспечить пустоту пересечения множеств, требуется усиленный вариант отделимости, назовем его истинной отделимостью множеств гиперплоскостью (В. Кли [2] называет такой тип отделимости "nicely seperation"). Геометрически истинная отделимость характеризуется таким взаимным расположением двух множеств и гиперплоскости, при котором одно из множеств лежит в алгебраически замкнутом полупространстве, порожденным гиперплоскостью, а другое принадлежит дополняющему его алгебраически открытому полупространству. Однако даже в конечномерных пространствах и, более того, на плоскости существуют непересекающиеся выпуклые множества, которые не могут быть истинно отделены никакой гиперплоскостью. Например, непересекающиеся выпуклые множества $A=\left\{\left(x_{1}, x_{2}\right) \in \mathbb{R}^{2} \mid x_{1}<0, x_{2} \leq 0\right\}$ и $B=\left\{\left(x_{1}, x_{2}\right) \in \mathbb{R}^{2} \mid x_{2} \geq \max \left\{0,-x_{1}^{3}\right\}\right.$ отделимы гиперплоскостью $H=\left\{\left(x_{1}, x_{2}\right) \in \mathbb{R}^{2} \mid x_{2}=0\right\}$, но их нельзя истинно отделить никакой гиперплоскостью.

Таким образом, существует разрыв между необходимыми и достаточными условиями неперескаемости выпуклых множеств, формулируемыми в терминах отделимости выпуклых множеств гиперплоскостями. Это обстоятельство находит отражение и в различных приложениях классических теорем об отделимости, в частности в теории оптимизации. Продемонстрируем это на примере задач выпуклого программирования.

Оптимальность допустимых решений в задачах выпуклого программирования может быть охарактеризована геометрически как условие непересечения двух выпуклых множеств, связанных с исследуемым допустимым решением. Применение классических теорем об отделимости выпуклых множеств гиперплоскостями позволяет получить из этого геометрического критерия как необходимые, так и достаточные условия оптимальности, между которыми, однако, существует разрыв (в необходимом условии оптимальности гарантируется лишь неотрицательность множителя Лагранжа, соответствующего целевому функционалу, тогда как в достаточном условии требуется его положительность). Критерий оптимальности Куна - Таккера справедлив для решений лишь тех задач задач выпуклого программирования, которые удовлетворяют дополнительному условию регулярности Слейтера. По существу, это условие выделяет те задачи выпуклого программирования, в которых соответствующие выпуклые множества в геометрическом критерии расположены так, что любая отделяющая их гиперплоскость является и истинно отделяющей. Благодаря этому условие оптимальности, которое в общем случае является лишь достаточным, становится и необходимым. Естественно, что достигается это за счет существенного сужения класса рассматриваемых задач выпуклого программирования.

Подобная ситуация возникает и при исследовании выпуклых задач векторной оптимизации. Любая минимальная точка выпуклого подмножества упорядоченного векторного пространства доставляет минимум на этом множестве некоторому положительному линейному функционалу. Однако не всякая точка, доставляющая минимум положительному линейному функционалу, является минимальной относительно заданного на векторном пространстве упорядочения. Для минимальности любой такой точки надо потребовать, чтобы минимизируемый линейный функционал был сильно положительным.

В 1930-е гг. прошлого века Ш. Какутани [3] и Дж. Тьюки [4] независимо доказали, что непрересекаемость двух выпуклых подмножеств векторного пространства эквивалентна их отделимости полупространствами (из более доступных источников см. [5, с. 23]). 
Напомним, что полупространствами называются такие выпуклые множества, дополнения которых также выпуклы.

Теорема (Какутани - Тьюки). Пусть $A$ и $B$ - выпуклые множества из векторного пространства $X$. Для того чтобы $A \cap B=\varnothing$, необходимо и достаточно, чтобы в $X$ существовало полупространство $H \subset X$ такое, что $A \subset H$, а $B \subset X \backslash H$.

Нетрудно видеть, что алгебраически замкнутые и алгебраически открытые полупространства, порождаемые гиперплоскостями, образуют собственное подсемейство в семействе всех полупространств. В силу этого теорема Какутани - Тьюки является прямым обобщением классических теорем об отделимости выпуклых множеств гиперплоскостями. Несмотря на общность, теорема Какутани - Тьюки не нашла достаточно широкого применения в анализе. Одна из возможных причин такого положения - отсутствие аналитического варианта этой теоремы. Для того чтобы сформулировать теорему Какутани - Тьюки аналитически, фактически, следует найти аналитическое представление произвольных полупространств. Решение этой проблемы в конечномерных векторных пространствах было дано Х.-Э. Мартинец-Легазом и И. Зингером [6] (см. ниже теорему 1).

Альтернативный подход, позволивший получить аналитическое представление полупространств в бесконечномерных векторных пространствах, был развит в работах [7-13]. Для этих целей был введен новый класс вещественнозначных функций, названных ступенчатоаффинными, и было показано, что функции этого класса двойственны полупространствам, причем для алгебраически замкнутых и алгебраически открытых полупространств эта двойственность совпадает с известной двойственностью, существующей между аффинными функциями и гиперплоскостями. Это позволило сформулировать и доказать аналитический вариант теоремы Какутани - Тьюки об отделимости выпуклых множеств полупространствами в произвольных вещественных векторных пространствах в виде теоремы об отделимости выпуклых множеств ступенчато-аффинными функциями (см. ниже предложение 4). В качестве приложений полученного аналитического варианта теоремы Какутани - Тьюки установлены критерий минимальности решений выпуклых задач векторной оптимизации и критерий оптимальности решений задач выпуклого программирования без каких-либо предположений о регулярности задачи. Если же задача выпуклого программирования удовлетворяет условию регулярности Слейтера, то установленный критерий совпадает с критерием Куна - Таккера.

Интерес к данной тематике по-прежнему сохраняется, о чем свидетельствуют появляющиеся в печати работы (см., например, [14-16]). Так, в статье [14] в рамках конечномерных пространств рассматриваются вопросы, связанные со специальным видом (open separation) отделимости выпуклых множеств полупространствами, при этом авторы существенно используют более ранние результаты из [6]. В то же время анализ работ [15;16], опубликованных в весьма авторитетных международных журналах, показывает, что их авторам (и не только им) не известны во многом окончательные результаты из $[2 ; 6 ; 8-13 ; 17-20]$. Как следствие, представленные в [15] результаты, касающиеся геометрической структуры конических полупространств в конечномерных пространствах, повторяют (без ссылок на первоисточники) уже известные результаты из $[6 ; 9 ; 17 ; 19 ; 20]$, а выводы из [16] о структуре бесконечномерных полупространств вовсе содержит ошибочные утверждения (об этом несколько подробнее будет сказано ниже).

Настоящая статья написана под влиянием работ [15;16] и имеет своей целью привлечь внимание исследователей к данной тематике, известным в ней результатам и некоторым открытым вопросам; при этом изложение ведется в бесконечномерных действительных векторных пространствах.

Исследования по данной тематики, начатые автором в семидесятые годы прошлого века, в значительной мере были стимулированы и неизменно поддерживались (тогда и позднее) Александром Борисовичем Куржанским, за что автор искренне ему благодарен. 


\section{1. Ступенчато-аффинные функции}

Здесь и всюду далее $X$ - вещественное векторное пространство, $\mathcal{A}(X)$ - векторное пространство, элементами которого являются аффинные функции, определенные на $X$. Ниже будем использовать то, что $\mathcal{A}(X)=L(X) \times \mathbb{R}$, где $L(X)$ - векторное пространство линейных функций на $X$. Если $f \in \mathcal{A}(X)$, то соответствующую ей пару из $L(X) \times \mathbb{R}$ будем обозначать через $\left(l_{f}, \alpha_{f}\right)$, тогда $f: x \rightarrow l_{f}(x)+\alpha_{f}$.

Для любого семейства $\mathcal{F} \subset \mathcal{A}(X)$, совершенно (линейно) упорядоченного ${ }^{2}$ некоторым отношением $\preccurlyeq \mathcal{F}$, и любого $f \in \mathcal{F}$ определим множества

$$
E_{f}:=\{x \in X \mid \bar{f}(x)=0 \text { для всех } \bar{f} \in \mathcal{F} \text { таких, что } \bar{f} \preccurlyeq \mathcal{F} f, \bar{f} \neq f\}
$$

и

$$
\widehat{E}_{f}:=\{x \in X \mid \bar{f}(x)=0 \text { для всех } \bar{f} \in \mathcal{F} \text { таких, что } \bar{f} \preccurlyeq \mathcal{F} f\} ;
$$

если $f$ является наименьшим (первым) элементом в $\mathcal{F}$, то положим $E_{f}=X$.

Нетрудно убедиться, что $E_{f}$ и $\widehat{E}_{f}$ являются аффинными многообразиями в $X$.

О п р е д е л е н и е 1 . Будем говорить, что семейство $\mathcal{F} \subset \mathcal{A}(X)$ образует кортеж аффинных функиий на $X$, если

(a) $\mathcal{F}$ совершенно упорядочено некоторым отношением $\preccurlyeq \mathcal{F}$;

(б) для каждой функции $f \in \mathcal{F}$ соответствующее ей аффинное многообразие $E_{f}$ является непустым и $f\left(E_{f}\right)=\mathbb{R}$;

(в) для каждого $x \in X$ либо подсемейство $\mathcal{F}_{x}:=\{f \in \mathcal{F} \mid f(x) \neq 0\}$ пусто, либо в $\mathcal{F}_{x}$ существует наименьший (относительно $\preccurlyeq \mathcal{F}$ ) элемент $f_{x}$.

Порядковый тип ${ }^{3}$ совершенно упорядоченного множества $(\mathcal{F}, \preccurlyeq \mathcal{F})$ будем называть рангом кортежа $\mathcal{F}$.

Отметим, что для любого кортежа аффинных функций $\mathcal{F} \subset \mathcal{A}(X)$ условие (б) эквивалентно условию

(б') для каждой функции $f \in \mathcal{F}$ существует точка $x \in X$ такая, что $f=f_{x}$ или, другими словами, каждая функция $f \in \mathcal{F}$ является наименьшим элементом подсемейства $\mathcal{F}_{x}$ для некоторого $x \in X$.

Кортеж аффинных функций $\mathcal{F}$ будем называть кортежем линейных функиий, если каждая функция $f \in \mathcal{F}$ является фактически линейной.

Из свойства (б) кортежа $\mathcal{F}$ следует, что $l_{f} \neq 0$ для любой функции $f \in \mathcal{F}$, т. е. кортеж аффинных функций не содержит константных функций, а кортеж линейных функций не содержит нулевую функцию.

Кортеж аффинных функций $\mathcal{F}$ будем называть регулярным (сингулярным), если аффинное подпространство

$$
E_{\mathcal{F}}=\{x \in X \mid f(x)=0 \quad f \in \mathcal{F}\}
$$

является непустым (пустым).

\footnotetext{
${ }^{2}$ Бинарное отношение $\preccurlyeq$, определенное на множестве $Z$, называется отношением совершенного (или линейного) порядка, если оно является полным отношением частичного порядка.

${ }^{3}$ Говорят, что совершенно упорядоченные множества $(U, \preccurlyeq U)$ и $(V, \preccurlyeq V)$ имеют один и тот же порядковый тип, если между ними может быть установлено взаимно однозначное соответствие, сохраняющее порядок. Два совершенно упорядоченных множества, имеющих одинаковый порядковый тип, являются равномощными. Более того, два конечных совершенно упорядоченных множества имеют один и тот же порядковый тип, если и только если они равномощны, при этом их порядковый тип отождествляется с числом их элементов. Бесконечные совершенно упорядоченные множества могут быть равномощными и иметь различный порядковый тип. Например, множество натуральных чисел $\mathbb{N}$, множество целых чисел $\mathbb{Z}$ и множество рациональных чисел $\mathbb{Q}$ имеют одну и ту же мощность $\aleph_{0}$ и в то же время порядковые типы всех трех множеств различны.
} 
Семейства линейных функций, удовлетворяющие условиям (а) и (в) впервые были введены и рассматривались В.Кли $[17 ; 18]$ в связи с аналитическим представлением семипространств (максимальных выпуклых множеств, не содержащих заданную точку). Термин кортеж линейных функций для таких семейств был введен В. В. Гороховиком $[7 ; 8]$. Кортежи аффинных функций были впервые введены в $[12 ; 13 ; 20]$. Данное здесь определение кортежа аффинных функций отличается от определения, приведенного ранее в $[12 ; 13]$, дополнительным условием (б), что позволяет избежать в дальнейшем необходимости выделять из всех кортежей несократимые кортежи.

Каждому кортежу аффинных функций $\mathcal{F}$ поставим в соответствие семейство линейных функций

$$
\mathcal{L}(\mathcal{F}):=\left\{l \in L(X) \mid \text { существует } f \in \mathcal{F} \text { такой, что } l=l_{f}\right\} \text {. }
$$

Будем считать, что $\mathcal{L}(\mathcal{F})$ упорядочено так же, как и $\mathcal{F}$, т. е. $l_{f_{1}} \preccurlyeq \mathcal{L}(\mathcal{F}) l_{f_{2}} \Longleftrightarrow f_{1} \preccurlyeq \mathcal{F} f_{2}$.

Покажем, что семейство $\mathcal{L}(\mathcal{F})$ является кортежем линейных функций. Действительно, условие (а) из определения 1 выполнено, так как $(\mathcal{L}(\mathcal{F}), \preccurlyeq \mathcal{L}(\mathcal{F}))$ - совершенно упорядоченное семейство. Кроме того, векторное пространство $E_{l_{f}}$ является параллельным непустому аффинному подпространству $E_{f}$ и, следовательно, также является непустым, при этом $l_{f}\left(E_{l_{f}}\right)=$ $f\left(E_{f}\right)=\mathbb{R}$. Это доказывает справедливость условия (б) для семейства $\mathcal{L}(\mathcal{F})$. Для доказательства условия (в) предположим, что $(\mathcal{L}(\mathcal{F}))_{x} \neq \varnothing$ для некоторого $x \in X$. Выберем произвольный $l_{f} \in(\mathcal{L}(\mathcal{F}))_{x}$. Поскольку для любого $a \in \widehat{E}_{f}$ имеем $l_{f^{\prime}}(a)=-\alpha_{f^{\prime}}$ для всех $f^{\prime} \in \mathcal{F}, f^{\prime} \preccurlyeq \mathcal{F} f$, то $l_{f^{\prime}}(x)=l_{f^{\prime}}(x+a)-l_{f^{\prime}}(a)=f^{\prime}(x+a)$ для всех $f^{\prime} \in \mathcal{F}, f^{\prime} \preccurlyeq \mathcal{F} f$. Значит, $f(x+a)=l_{f}(x) \neq 0$ и, следовательно, $\mathcal{F}_{x+a} \neq \varnothing$, причем $f \in \mathcal{F}_{x+a}$. Так как $f_{x+a} \preccurlyeq \mathcal{F} f$, то $l_{f_{x+a}}(x)=f_{x+a}(x+a) \neq 0$, что влечет $l_{f_{x+a}} \in(\mathcal{L}(\mathcal{F}))_{x}$. Из равенства $l_{f^{\prime}}(x)=f^{\prime}(x+a)$ для $f^{\prime} \preccurlyeq \mathcal{F} f$ следует также, что $l_{f^{\prime}}(x)=0$ для всех $f^{\prime} \preccurlyeq \mathcal{F} f_{x+a}, f^{\prime} \neq f_{x+a}$. Итак, $l_{f_{x+a}}$ является наименьшим элементом в $(\mathcal{L}(\mathcal{F}))_{x}$. Это завершает доказательство того, что $\mathcal{L}(\mathcal{F})$ есть кортеж линейных функций.

Всюду ниже будем называть $\mathcal{L}(\mathcal{F})$ кортежем линейных функиий, соответствующим кортежу аффинных функиий $\mathcal{F}$.

Из свойства (в) кортежа следует, что кортеж линейных функций, соответствующий любому кортежу аффинных функций, является линейно независимым семейством в векторном пространстве $L(X)$ и, следовательно, любой кортеж аффинных функций $\mathcal{F}$ на конечномерном векторном пространстве конечен, причем число его элементов не превосходит коразмерности аффинного пространства $E_{\mathcal{F}}$.

Любое вполне упорядоченное линейно независимое семейство линейных функций является кортежем. В частности, любое конечное упорядоченное семейство линейных функций является кортежем в том и только том случае, когда оно линейно независимо.

О п р е д е л е н и е $2[12 ; 13]$. Вещественнозначную функцию $u: X \rightarrow \mathbb{R}$ будем называть ступенчато-аффинной (соответственно ступенчато-линейной), если существует кортеж аффинных функций (соответственно кортеж линейных функций) $\mathcal{F}$ такой, что $u(x)=u_{\mathcal{F}}(x)$ для всех $x \in X$, где

$$
u_{\mathcal{F}}(x)=\left\{\begin{array}{cc}
0, & \text { если } \mathcal{F}_{x}=\varnothing, \\
f_{x}(x), & \text { если } \mathcal{F}_{x} \neq \varnothing .
\end{array}\right.
$$

Ранг ступенчато-аффинной функиии $u: X \rightarrow \mathbb{R}$ будем считать равным рангу порождающего ее кортежа $\mathcal{F}$, т. е. такого кортежа $\mathcal{F}$, что $u=u_{\mathcal{F}}$.

Если кортеж аффинных функций $\mathcal{F}=\left\{f_{1}, f_{2}, \ldots, f_{m}\right\}$ конечен, то соотношение (1.1) может быть записано следующим образом:

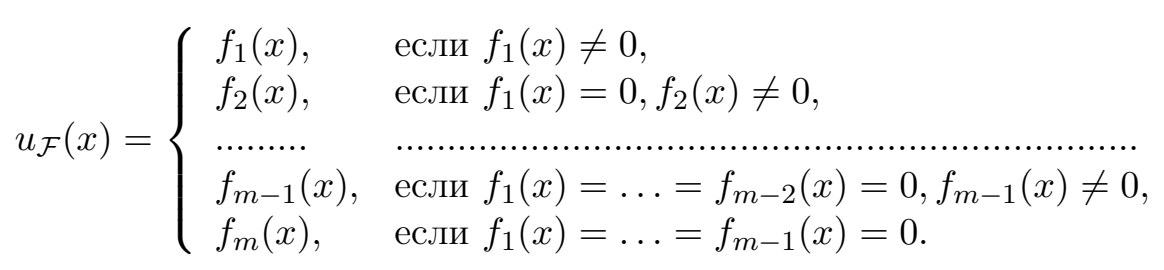


Любая ненулевая линейная функция является ступенчато-линейной, тогда как аффинная функция ступенчато-аффинна в том и только том случае, когда она не равна константе. Из однородности линейных функций следует, что каждая ступенчато-линейная функция также однородна.

Пусть $X=\mathbb{R}^{n}$. Так как пространство $L\left(\mathbb{R}^{n}\right)$ линейных функций, определенных на $\mathbb{R}^{n}$, изоморфно $\mathbb{R}^{n}$, то любой кортеж $\mathcal{F}=\left\{l_{1}, l_{2}, \ldots, l_{k}\right\}$ линейных функций из $L\left(\mathbb{R}^{n}\right)$ может быть отождествлен с упорядоченным линейно независимым семейством векторов $\Lambda=\left\{\lambda_{1}, \ldots, \lambda_{k}\right\}$ из $\mathbb{R}^{n}$, которое будем называть кортежем векторов в $\mathbb{R}^{n}$. Если векторы, образующие кортеж $\Lambda$, взаимно ортогональны, то такой кортеж векторов $\Lambda$ будем называть ортогональным.

Предложение 1. Ненулевая функция $w: \mathbb{R}^{n} \rightarrow \mathbb{R}$ является ступенчато-линейной тогда и только тогда, когда на $\mathbb{R}^{n}$ существует единственным образом определенный ортогональный кортеж векторов $\Lambda=\left\{\lambda_{1}, \ldots, \lambda_{k}\right\}, 1 \leq k \leq n$, такой, что

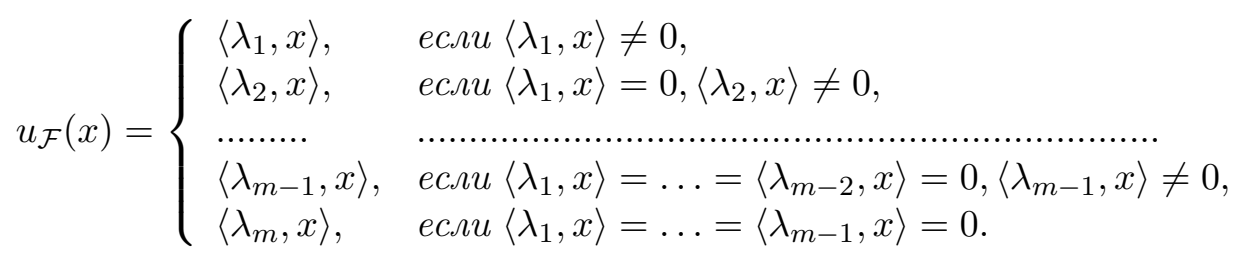

О п р е д е л е н и е 2 . Ступенчато-аффинную функцию $u: X \rightarrow \mathbb{R}$ будем называть регулярной (соответственно сингулярной), если кортеж, который порождает ее является регулярным (соответственно сингулярным).

Заметим, что любая сингулярная ступенчато-аффинная функция отлична от нуля на всем пространстве $X$. Из этого следует, что любая ступенчато-линейная функция, рассматриваемая как ступенчато-аффинная, является регулярной. Более того, справедливо следующее утверждение.

Предложение 2. Ступенчато-аффинная функиия $u: X \rightarrow \mathbb{R}$ является регулярной тогда и только тогда, когда существуют ступенчато-линейная функиия $c: X \rightarrow \mathbb{R}$ и точка $a \in X$ maкuе, чmo

$$
u(x)=c(x-a) \partial л я в \text { всех } x \in X .
$$

Д о к а з а т е л ь с т в о. Пусть $u: X \rightarrow \mathbb{R}$ - регулярная ступенчато-аффинная функция и пусть $\mathcal{F}-$ кортеж аффинных функций, порождающий $u$. Тогда аффинное многообразие $E_{\mathcal{F}} \neq \varnothing$, при этом для каждого $a \in E_{\mathcal{F}}$ и для каждого $f \in \mathcal{F}$ выполняется равенство $f(a)=$ $l_{f}(a)+\alpha_{f}=0$. Из этого следует, что $f(x)=l_{f}(x)+\alpha_{f}=l_{f}(x-a)$ для всех $x \in X$ и для всех $f \in \mathcal{F}$. Значит, $(\mathcal{L}(\mathcal{F}))_{x-a}=\left\{l_{f} \in L(X) \mid f \in \mathcal{F}_{x}\right\}$ и, следовательно, $u_{\mathcal{F}}(x)=u_{\mathcal{L}(\mathcal{F})}(x-a)$ для всех $x \in X$.

Обратное утверждение следует из того, что для любого кортежа линейных функций $\mathcal{L}$ и любой точки $a \in X$ семейство $\mathcal{F}=\{x \rightarrow l(x-a) \mid l \in \mathcal{L}\}$ является регулярным кортежем аффинных функций.

\section{2. Полупространства}

Подмножество $H$ действительного векторного пространства $X$ называется nолупространством в $X$, если $H$ и его дополнение $X \backslash H$ являются выпуклыми подмножествами в $X$. Выпуклые конусы, дополнения к которым также являются выпуклыми конусами, называются коническими полупространствами.

Непосредственно из определения следует, что дополнение любого полупространства также является полупространством. В частности, пустое множество $\varnothing$ и все пространство $X$ являются взаимно дополняющими друг друга несобственными полупространствами. Полупространства, отличные от пустого множества и всего пространства $X$, будем называть собственными. 
Наиболее широко распространенную пару взаимно дополняющих собственных полупространств образуют подмножества

$$
H_{<}(l, \alpha):=\{x \in X \mid l(x)<\alpha\} \quad \text { и } H_{\geq}(l, \alpha):=\{x \in X \mid l(x) \geq \alpha\},
$$

где $l: X \mapsto \mathbb{R}(l \neq 0)$ - ненулевой линейный функционал на $X$, а $\alpha \in \mathbb{R}$ - вещественное число.

Другой известный пример взаимно дополняющих собственных полупространств задается семипространством и его дополнением.

Напомним, что подмножество $S \subset X$ называется семипространством, порожденным точкой $a \in X$ (или просто семипространством в точке $a \in X$ ), если $S$ является максимальным выпуклым множеством, не содержащим точку $a$.

Семипространства были введены П.К. Хаммером [21]. Им было показано, что совокупность всех семипространств образует наименьшую базу выпуклости в $X$ (каждое выпуклое множество из $X$ есть пересечение некоторого семейства семипространств). В. Кли [17;18] изучил геометрическую структуру семипространств и использовал полученные при этом результаты для аналитического представления и классификации семипространств. М. Лассак и А. Прушински [22;23] распространили класс семипространств, порожденных точками, до класса обобщенных семипространств, порожденных аффинными многообразиями. В частности, введенное выше полупространство $H_{<}(l, \alpha)$ является обобщенным семипространством, порожденным гиперплоскостью $H_{=}(l, \alpha):=\{x \in X \mid l(x)=\alpha\}$.

Ниже, говоря о полупространствах, будем иметь в виду собственные полупространства.

В конечномерных векторных пространствах геометрическая структура и аналитическое представление полупространств были исследованы в работах $[6 ; 9 ; 19 ; 20]$. Наиболее исчерпывающее исследование конечномерных полупространств было проведено Х.-Э. Мартинец-Легазом и И. Зингером [6;24]. В частности, ими была осуществлена классификация конечномерных полупространств, основанная на их аналитическом представлении, при этом каждый класс полупространств характеризуется двумя инвариантами - типом $\tau(H)$ и рангом $r(H)$ полупространства $H$.

Теорема 1 [6;24]. Пусть $X$ - конечномерное векторное пространство. Собственное подмножество $H \subset X$ является полупространством в $X$ тогда и только тогда, когда найдутся натуральное число $m \in\{1,2, \ldots, \operatorname{dim} X\}$, сюрьективный линейный оператор $A: X \mapsto \mathbb{R}^{m} u$ вектор $\alpha:=\left\{\alpha_{1}, \alpha_{2}, \ldots, \alpha_{m}\right\} \in \mathbb{R}^{m}$ такие, что либо

$$
H=\left\{x \in X \mid A x<_{L} \alpha\right\},
$$

либо

$$
H=\left\{x \in X \mid A x \leq_{L} \alpha\right\} .
$$

Здесъ $<_{L} u \leq_{L}$ обозначают соответственно отношение строгого лексикографического порядка и отношение лексикографического порядка на $\mathbb{R}^{m}$.

Напомним, что для любых $u=\left(u_{1}, u_{2}, \ldots, u_{m}\right)$ и $v=\left(v_{1}, v_{2}, \ldots, v_{m}\right)$ соотношения $u<_{L} v$ и $u \leq_{L} v$ определяются следующим образом:

$$
\begin{gathered}
u<_{L} v \Longleftrightarrow u \neq v \text { и } u_{k}<v_{k} \text { для } k=\min \left\{i \in\{1,2, \ldots, m\} \mid u_{i} \neq v_{i}\right\}, \\
u \leq_{L} v \Longleftrightarrow u=v \text { или } u<_{L} v .
\end{gathered}
$$

В классификации Мартинец-Легаза и Зингера тип $\tau(H)$ полупространства $H$ отождествляется с типом неравенства в аналитическом представлении, заданном либо (2.1), либо (2.2), т. е. $\tau(H) \in\left\{<_{L}, \leq_{L}\right\}$, а ранг $r(H)$ принимается равным рангу линейного оператора $A: X \mapsto \mathbb{R}^{m}$, т. е. $r(H)=m$. Согласно классификации Х.-Э. Мартинец-Легаза и И. Зингера два полупространства $H_{1}$ и $H_{2}$ принадлежат одному и тому же классу, если $\tau\left(H_{1}\right)=\tau\left(H_{2}\right)$ и $r\left(H_{1}\right)=r\left(H_{2}\right)$, т. е. если они имеют один и тот же тип и ранг. 
В работах [10-13] понятие типа и ранга было распространено на бесконечномерные полупространства.

Понятие типа полупространства основано на следующей порядковой характеристике полупространств.

Предложение 3 [10;13]. Подмножество Н векторного пространства $X$ является полупространством в том и только том случае, когда пара подмножеств $(X \backslash H, H)$ образует сечение пространства $X$ относительно полного отношения предпорядка $\preceq_{H}$, определенного на $X$ условием

$$
x \preceq_{H} y \Longleftrightarrow y-x \in 0^{+} H,
$$

где $0^{+} H:=\{h \in X \mid x+t h \in H$ для всех $x \in H u t \geq 0\}-$ рецессивный конус множества $H$.

Нетрудно убедиться, что подмножество $H \subset X$ является полупространством в $X$ в том и только том случае, когда его рецессивный конус $0^{+} H$ является коническим полупространством в $X$. Так как $0^{+} H-$ выпуклый конус, то ${ }_{H}$ является отношением предпорядка ${ }^{4}$ на $X$, согласованным с алгебраическими операциями пространства $X$, а из того, что $X=0^{+} H \cup\left(-0^{+} H\right)$, следует, что отношение $\preceq_{H}$ является полным.

В [10;13] тип полупространства $H \subset X$ отождествляется с типом порождаемого им сечения $(X \backslash H, H)$ пространства $X$ по отношению $\preceq_{H}$.

Напомним, что под сечением $(A, B)$ множества $V$, упорядоченного полным отношением предпорядка $\preccurlyeq$, понимается такое разбиение $V$ на два непересекающиеся подмножества $A$ и $B$, что $u \preccurlyeq v, v \npreceq u$ для любых $u \in A$ и $v \in B$. Подмножество $A$ называется нижним классом сечения $(A, B)$, а подмножество $B$ - верхним классом.

Известно (см., например, [25]), что в множествах, упорядоченных полным отношением предпорядка, могут существовать четыре типа сечений: скачок (в нижнем классе сечения есть наибольший элемент, а в верхнем классе есть наименьший), нижнее дедекиндово сечение (в нижнем классе сечения есть наибольший элемент, а в верхнем классе нет наименьшего элемента), верхнее дедекиндово сечение (в нижнем классе сечения нет наибольшего элемента, а в верхнем классе есть наименьший элемент) и щель (в нижнем классе сечения нет наибольшего элемента, а в верхнем классе нет наименьшего элемента).

В [10;13] показано, что среди сечений $(X \backslash H, H)$ векторного пространства $X$, порожденных полупространствами $H$, а) не существует скачков; б) в любом векторном пространстве существуют верхние и нижние дедекиндовы сечения; в) в конечномерных векторных пространствах не существует сечений-щелей; г) в каждом бесконечномерном векторном пространстве существуют полупространства, порождающие сечения-щели.

Полупространства, порождающие верхнее (соответственно нижнее) дедекиндово сечение, названы заостренными (соответственно незаостренными) дедекиндовыми полупространствами, а полупространства, порождающие сечения-щели, - недедекиндовыми полупространствами.

Таким образом, в каждом бесконечномерном векторном пространстве существуют три типа полупространств: два (заостренное и незаостренное) дедекиндовы и одно недедекиндово.

\footnotetext{
${ }^{4}$ Говорят, что отношение предпорядка (рефлексивное и транзитивное бинарное отношение) $\preceq$, заданное на векторном пространстве $X$, согласовано с алгебраическими операциями пространства $X$, если$$
x \preceq y \Longrightarrow x+z \preceq y+z \forall x, y, z \in X
$$

и

$$
x \preceq y \Longrightarrow \lambda x \preceq \lambda y \forall x, y \in X, \lambda>0 .
$$
}

Если $\preceq$ - согласованное отношение предпорядка, то

$$
x \preceq y \Longleftrightarrow y-x \in P_{\preceq},
$$

где $P_{\preceq}:=\{x \in X \mid 0 \preceq x\}-$ (выпуклый) конус положительных элементов отношения $\preceq$. 
В то же время в конечномерных векторных пространствах существуют только два типа полупространств: заостренное и незаостренное дедекиндовы. В классификации конечномерных полупространств, принадлежащей Х.-Э. Мартинец-Легазу и И. Зингеру, заостренным дедикиндовым полупространствам соответствует тип $\leq_{L}$, а незостренным дедекиндовым полупространствам - тип $<_{L}$. Если $H$ - заостренное (незаостренное) дедикиндово полупространство, то его дополнение $X \backslash H$ является незаостренным (заостренным) дедекиндовым, если же $H-$ недедекиндово полупространство, то $X \backslash H$ также недедекиндово полупространство. Любое коническое полупространство является заостренным или незаостренным дедекиндовым полупространством. Алгебраически замкнутое полупространство является заостренным дедекиндовым, а алгебраически открытое полупространство - незаостренным дедекиндовым. Семейство всех незаостренных дедекиндовых полупространств совпадает с семейством обобщенных семипространств.

Перейдем к определению ранга бесконечномерных полупространств. Для этого воспользуемся тем, что, как это показано в [12;13], любое (конечномерное или бесконечномерное) полупространство может быть задано аналитически при помощи ступенчато-аффинных функций. Если быть более точным, то справедливы следующие утверждения.

Предложение 4. Пусть $H$ - собственное подмножество векторного пространства $X$.

а) $Н$ является заостренным дедекиндовым полупространством в $X$ тогда и только тогда, когда существует регулярнал ступенчато-аффинная функиия $u_{H}: X \rightarrow \mathbb{R}$ такал, что

$$
H=\left\{x \in X \mid u_{H}(x) \leq 0\right\} ;
$$

б) тогда, когда существует регулярная ступенчато-афбинная функция $u_{H}: X \rightarrow \mathbb{R}$ такал, что

$$
H=\left\{x \in X \mid u_{H}(x)<0\right\} ;
$$

в) $H$ является недедекиндовым полупространством в $X$ тогда и толъко тогда, когда существует сингулярная ступенчато-афбинная функиия $u_{H}: X \rightarrow \mathbb{R}$ такал, что

$$
H=\left\{x \in X \mid u_{H}(x) \leq 0\right\}=\left\{x \in X \mid u_{H}(x)<0\right\} ;
$$

г) Н является коническим заостренным (незаостренным) дедекиндовым полупространством в $X$ тогда и только тогда, когда существует ступенчато-линейная функиия $w_{H}$ : $X \rightarrow \mathbb{R}$ такал, что

$$
\begin{gathered}
H=\left\{x \in X \mid w_{H}(x) \leq 0\right\} \\
\left(H=\left\{x \in X \mid w_{H}(x)<0\right\}\right) .
\end{gathered}
$$

Равенство двух представлений $H=\left\{x \in X \mid u_{H}(x)<0\right\}$ и $H=\left\{x \in X \mid u_{H}(x) \leq 0\right\}$ для недедекиндовых полупространств обусловлено тем, что сингулярные ступенчато-аффинные функции отличны от нуля на всем пространстве $X$.

Как гиперплоскость может быть задана различными аффинными функциями, так и каждое полупространство $H$ может быть задано аналитически различными ступенчато-аффинными функциями $u_{H}: X \rightarrow \mathbb{R}$. Общим для всех ступенчато-аффинных функций, задающих аналитическое представление одного и того же полупространства $H$, является то, что они имеют один и тот же ранг. Это свойство приводит к следующему определению ранга полупространства.

Ранг полупространства $H$ отождествляется с рангом ступенчато-аффинной функции $u_{H}$ : $X \rightarrow \mathbb{R}$, задающей аналитическое представление $H$.

Нетрудно видеть, что ранг алгебраически замкнутых и алгебраически открытых полупространств равен единице. 
В конечномерных пространствах приведенное выше определение ранга полупространства совпадает с определением ранга, данным в работах Х.-Э. Мартинец-Легаза и И. Зингера [6; 24].

Будем говорить, что два полупространства $H_{1}$ и $H_{2}$ принадлежат одному классу, если они имеют один и тот же тип и их ранги равны.

Поскольку ступенчато-аффинные функции $u$ и $-u$ имеют один и тот же ранг, то ранги полупространства $H$ и дополняющего его полупространства $X \backslash H$ также равны. Если $H-$ дедекиндово полупространство, то $H$ и $X \backslash H$ имеют различный тип и, следовательно, принадлежат различным классам, хотя имеют один и тот же ранг. Если же полупространство $H$ недедикиндово, то $H$ и $X \backslash H$ принадлежат одному классу, поскольку имеют один и тот же тип и равные ранги.

3 а м е ч а н и е 1 . Согласованное отношение предпорядка $\preceq$, определенное на векторном пространстве $X$, является полным в том и только том случае, когда соответствующий ему конус положительных элементов $P_{\preceq}$ есть заостренное коническое полупространство. Для согласованных отношений совершенного порядка и только для них конус $P_{\preceq}$ удовлетворяет, кроме того, дополнительному условию $P_{\preceq} \cap\left(-P_{\preceq}\right)=\{0\}$. Характеризуя геометрическое строение конусов положительных элементов $P_{\preceq}$, соответствующих согласованным отношениям совершенного порядка $\preceq$, определеным в сепарабельном гильбертовом пространстве, авторы работы [16] ошибочно полагают, что любой такой конус имеет непустую внутренность. На самом деле это верно лишь для весьма частного вида таких конусов, наряду с которыми в сепарабельном гильбертовом пространстве существуют такие заостренные конические полупространства $P_{\preceq}$ со свойством $P_{\preceq} \cap\left(-P_{\preceq}\right)=\{0\}$, ранг которых равен порядковому типу любого счетного совершенно упорядоченного множества, в частности существуют полупространства, ранг которых равен порядковому типу множества целых чисел. Естественно, что ошибочное исходное положение приводит авторов работы [16] к ошибочным выводам о геометрическом строении всего семейства конусов положительных элементов, соответствующих согласованным отношеним совершенного порядка, определенным на сепарабельном гильбертовом пространстве (см. теорему 3.5 из [16]).

\section{3. Аналитические критерии отделимости выпуклых множеств}

Основная цель настоящего раздела - получить эквивалентные теореме Какутани - Тьюки аналитические критерии непересекаемости выпуклых множеств, используя при этом в качестве аналитических средств, отделяющих непересекающиеся множества друг от друга, ступенчато-аффинные и ступенчато-линейные функции.

Начнем со следующего критерия отделимости выпуклых множеств полными согласованными отношениями предпорядка.

Теорема 2. Для того чтобы выпуклые множества $A$ и $B$ вещественного векторного пространства $X$ не пересекалисъ, необходимо и достаточно, чтобы на $X$ существовало полное согласованное отношение предпорядка $\preceq$ такое, что

$$
y \prec x \text { для всех } x \in A \text { и всех } y \in B \text {. }
$$

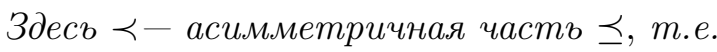

$$
y \prec x \Longleftrightarrow y \preceq x, x \npreceq y .
$$

Д о к а з а т е л ь с т в о. Достаточность следует из асимметричности отношения $\prec$.

Необходимость. Предположим, что $A \cap B=\varnothing$. По теореме Какутани - Тьюки существует полупространство $H \subset X$ такое, что $A \subset H$ и $B \subset X \backslash H$. Воспользуемся далее предложением 3, согласно которому подмножество $H \subset X$ является полупространством в $X$ тогда и только тогда, когда пара $(X \backslash H, H)$ является сечением пространства $X$ относительно полного 
согласованного отношения предпорядка $\preceq_{H}$. Из определения сечения получаем, что $y \prec_{H} x$ для всех $x \in H$ и всех $y \in X \backslash H$, а поскольку $A \subset H$ и $B \subset X \backslash H$, то $y \prec_{H} x$ для всех $x \in A$ и $y \in B$.

Далее нам понадобится следующее утверждение.

Предложение 5. Согласованное отношение предпорядка $\preceq$, определенное на $X$, является полным тогда и только тогда, когда существует ступенчато-линейная функиия $w: X \rightarrow \mathbb{R}$ такая, что

$$
x \preceq y \Longleftrightarrow y-x \in P_{\preceq} \Longleftrightarrow w(y-x) \geq 0 .
$$

Д о к а з а т е л ь с т в о. Как уже указывалось в замечании 1, согласованное отношение предпорядка $\preceq$, определенное на $X$, является полным тогда и только тогда, когда соответствующий ему конус положительных элементов $P_{\preceq}$ является коническим заостренным дедикиндовым полупространством. В силу предложения 4 для $P \preceq$ в этом случае существует ступенчато-линейная функция $w: X \rightarrow \mathbb{R}$ такая, что $P_{\preceq}=\{z \in X \mid w(z) \geq 0\}$. Следовательно,

$$
x \preceq y \Longleftrightarrow y-x \in P_{\preceq} \Longleftrightarrow w(y-x) \geq 0 .
$$

Используя предложение 4, получим в качестве следствия порядкового критерия из теоремы 2 критерий отделимости выпуклых множеств ступенчато-линейными функциями.

Теорема 3. Для того чтобы выпуклые множества $A$ и $B$ вещественного векторного пространства $X$ не пересекались, необходимо и достаточно, чтобы существовала ступенчато-линейная функиия $w: X \rightarrow \mathbb{R}$ такал, что

$$
w(x-y)>0 \text { для всех } x \in A \text { и всех } y \in B .
$$

Покажем далее, что для случая, когда множества $A$ и $B$ являются выпуклыми конусами в $X$, теорема 3 может быть несколько уточнена и приведена к более удобной для приложений форме, поскольку условия, которым в них подчинены отделяемые множества, в ней разделены.

Заметим, что если два выпуклых конуса не пересекаются, то, по крайней мере, один из них не содержит нулевой точки и, следовательно, является асимметричным. Поэтому предположение об асимметричности одного из конусов в условиях следующей теоремы не ограничивает на самом деле общности рассматриваемой ситуации.

Теорема 4. Пусть $K_{1}$ и $K_{2}$ - выпуклые конусы в векторном пространстве $X$, причем конус $K_{1}$ асимметричен. Тогда следующие утверждения эквивалентны:

а) $K_{1} \cap K_{2}=\varnothing$;

б) существует коническое заостренное полупространство $H$ такое, что $K_{1} \cap H=\varnothing$, $K_{2} \subset H$

в) существует ступенчато-линейная функиия $w: X \rightarrow \mathbb{R}$ такая, что

$$
w(x)>0 \text { для всех } x \in K_{1}, \quad w(x) \leq 0 \text { для всех } x \in K_{2} .
$$

Д о к а з а т е л ь с т в о. Докажем последовательно импликации а) $\Rightarrow$ б) $\Rightarrow$ в) $\Rightarrow$ a). Обозначим символом $\mathcal{C}\left(K_{1}\right)$ семейство, состоящее из таких выпуклых конусов $K \subset X$, которые не пересекаются с $K_{1}$. Нетрудно убедиться, что семейство $\mathcal{C}\left(K_{1}\right)$ индуктивно упорядочено отношением включения. Следовательно, в силу леммы Цорна (см., например, [26, с. 40]) семейство $\mathcal{C}\left(K_{1}\right)$ обладает максимальными элементами, причем для любого $K \in \mathcal{C}\left(K_{1}\right)$ в $\mathcal{C}\left(K_{1}\right)$ существует максимальный выпуклый конус, содержащий $K$. Так как $K_{2} \in \mathcal{C}\left(K_{1}\right)$, то для него тоже можно указать максимальный выпуклый конус $C \in \mathcal{C}\left(K_{1}\right)$ такой, что $K_{2} \subset C$. Из условия $0 \notin K_{1}$ и максимальности $C$ следует, что $0 \in C$. Поскольку $K_{1} \cap C=\varnothing$, то по теореме Какутани - Тьюки в $X$ существует полупространство $H$ такое, что $C \subset H$ и $K_{1} \subset X \backslash H$. Рассмотрим 
коническую оболочку полупространства $H$, которую обозначим через cone $H$. Из выпуклости $H$ следует, что cone $H$ есть выпуклый конус, причем $C \subset H \subset$ cone $H$. Условие $K_{1} \cap H=\varnothing$ влечет за собой (поскольку $K_{1}$ - асимметричный конус) соотношение $K_{1} \cap$ cone $H=\varnothing$ и, следовательно, cone $H \in \mathcal{C}\left(K_{1}\right)$. В силу максимальности $C$ заключаем, что $C=H=$ cone $H$. Таким образом, $C$ - заостренное коническое полупространство, удовлетворяющее условию б). Импликация а) $\Rightarrow$ б) доказана.

Чтобы доказать б) $\Rightarrow$ в), достаточно воспользоваться утверждением г) предложения 4 и тем, что $0 \in H$.

Так как импликация в) $\Rightarrow$ а) очевидна, то теорема доказана полностью.

Сформулируем и докажем наконец общую теорему об отделимости выпуклых множеств ступенчато-аффинными функциями.

Теорема 5. Для того чтобы выпуклые подмножества $A$ и В вещественного векторного пространства $X$ не пересекались, необходимо и достаточно, чтобы существовала такая ступенчато-аффинная функиия $u: X \rightarrow \mathbb{R}$, что либо

$$
u(x)>0 \text { для всех } x \in A, \quad u(y) \leq 0 \text { для всех } y \in B,
$$

либо

$$
u(x) \geq 0 \text { для всех } x \in A, \quad u(y)<0 \text { для всех } y \in B .
$$

Д о к а з а т е л ь с т в о. Необходимость. В силу теоремы Какутани - Тьюки, сформулированной во введении, выпуклые множества $A$ и $B$ не пересекаются в том и только том случае, когда в $X$ существует полупространство $H$ такое, что $A \subset H$ и $B \subset X \backslash H$. Из утверждений предложения 4 следует существование такой ступенчато-аффинной функции $u: X \rightarrow \mathbb{R}$, что либо $H=\{x \in X \mid u(x)>0\}$, либо $H=\{x \in X \mid u(x) \geq 0\}$.

Так как $A \subset H$, а $B \subset X \backslash H$, то легко видеть, что функция $u: X \rightarrow \mathbb{R}$ удовлетворяет либо условию (3.1), либо условию (3.2).

Достаточностъ. То, что из каждого условия (3.1) и (3.2) следует пустота пересечения множеств $A$ и $B$, проверяется непосредственно.

\section{4. Критерий оптимальности решений в выпуклой задаче векторной оптимизации}

В наиболее общем виде задача оптимизации может быть сформулирована следующим образом.

Пусть заданы некоторое множество $Q$ и отношение предпорядка $\preceq$, определенное на $Q$.

Точка $x^{0} \in Q$ называется $\preceq$-минимальной в $Q$ (минимальной в $Q$ относительно $\left.\preceq\right)$, если в $Q$ не существует точки $\bar{x} \in Q$ такой, что $\bar{x} \prec x^{0}$ (здесь $y \prec x \Longleftrightarrow y \preceq x, x \npreceq y$ ).

Множество $\preceq$-минимальных точек множества $Q$ будем обозначать символом $\operatorname{Min}(Q \mid \preceq)$.

Задача оптимизации, определенная парой $(Q, \preceq)$, состоит в нахождении для заданного множества $Q$ и определенного на нем отношения предпорядка $\preceq$ множества $\preceq$-минимальных элементов $\operatorname{Min}(Q \mid \preceq)$.

Множество $Q$ называется при этом множеством допустимых решений задачи оптимизации, отношение $\preceq-$ отношением предпочтения, а $\operatorname{Min}(Q \mid \preceq)-$ множеством оптимальных решений.

Если отношение предпочтения $\preceq$ обладает функцией полезности, т. е. если существует вещественнозначная функция $f: Q \rightarrow \mathbb{R}$ такая, что

$$
x \preceq y \Longleftrightarrow f(x) \leq f(y) \text { для любых } x, y \in Q,
$$

то $x^{0} \in \operatorname{Min}(Q \mid \preceq)$ тогда и только тогда, когда

$$
f\left(x^{0}\right)=\min _{x \in Q} f(x) .
$$


В этом случае задача оптимизации приобретает классический вид и заключается в минимизации на $Q$ вещественнозначной функции $f$.

Задача оптимизации $(Q, \preceq)$ называется задачей векторной оптимизации, если множество $Q$ - подмножество некоторого векторного пространства $X$, а отношение предпочтения $\preceq$ определено на всем $X$ и является согласованным с алгебраическими операциями.

Нетрудно убедиться в справедливости следующего утверждения.

Предложение 6. Для того чтобы $x^{0} \in Q$ было оптимальным решением в задаче векторной оптимизачии $(Q, \preceq)$, необходимо и достаточно, чтобъ

$$
\left(Q-\left\{x^{0}\right\}\right) \cap\left(-P_{\prec}\right)=\varnothing,
$$

где $P_{\prec}:=\{x \in X \mid 0 \prec x\}-$ конус строго положительных векторов отношения $\preceq$.

Задача векторной оптимизации $(Q, \preceq)$ называется выпуклой, если выпуклым является множество $\widehat{Q}:=\{x \in X \mid \exists y \in Q: y \preceq x\}$.

Говорят, что однородная функция $\varphi: X \rightarrow \mathbb{R}$ является сильно $\preceq$-положительной, если

$\varphi(x)>0$ для всех $x \in X$ таких, что $0 \prec x$ и $\varphi(x)=0$ для всех $x \in X$ таких, что $x \sim 0$,

где $x \sim 0 \Longleftrightarrow 0 \preceq x, x \preceq 0$.

Теорема 6. Для того чтобы $x^{0}$ было оптимальным решением выпуклой задачи векторной оптимизачии $(Q, \preceq)$, необходимо и достаточно, чтобы существовала сильно Қ-положительная ступенчато-линейная функиия $w: X \rightarrow \mathbb{R}$ такал, что

$$
w\left(x-x^{0}\right) \geq 0 \text { для всех } x \in Q .
$$

Д о к а з а т е л ь с т в о. Необходимостъ. Прежде всего отметим, что для выпуклых задач векторной оптимизации критерий минимальности (4.1) эквивалентен условию

$$
\operatorname{cone}\left(\widehat{Q}-\left\{x^{0}\right\}\right) \cap\left(-P_{\prec}\right)=\varnothing,
$$

где сопе $\left(\widehat{Q}-\left\{x^{0}\right\}\right)-$ коническая оболочка множества $\widehat{Q}-\left\{x^{0}\right\}$. Так как множество $\widehat{Q}$ выпукло, то cone $\left(\widehat{Q}-\left\{x^{0}\right\}\right)$ - выпуклый конус. Поскольку $P_{\prec}-$ асимметричный выпуклый конус, то, применяя теорему 4 , получим, что условие (4.3) равносильно существованию ступенчатолинейной функции $w: X \rightarrow \mathbb{R}$ такой, что

$$
w(x)<0 \text { для всех } x \in-P_{\prec}
$$

и

$$
w(x) \geq 0 \text { для всех } x \in \operatorname{cone}\left(\widehat{Q}-\left\{x^{0}\right\}\right) .
$$

Поскольку $0 \in Q-\left\{x^{0}\right\}$, то $P_{\preceq}:=\{x \in X \mid 0 \preceq x\} \subset \operatorname{cone}\left(\widehat{Q}-\left\{x^{0}\right\}\right)$. Поэтому из (4.5) имеем

$$
w(x) \geq 0 \text { для всех } x \in P_{\preceq} .
$$

Условия (4.4) и (4.6) показывают, что ступенчато-линейная функция $w: X \rightarrow \mathbb{R}$ сильно $\preceq$-положительна; кроме того, из условия (4.5) следует (4.2). Необходимость доказана.

Достаточность. Любая сильно Љ-положительная ступенчато-линейная функция $w$ : $X \rightarrow \mathbb{R}$ удовлетворяет условиям (4.4) и (4.6), а условия (4.6) и (4.2) влекут за собой (4.5). Следовательно, при выполнении условий достаточной части теоремы имеют место неравенства (4.4) и (4.5), равносильные (4.3), а (4.3) в свою очередь эквивалентно (4.1).

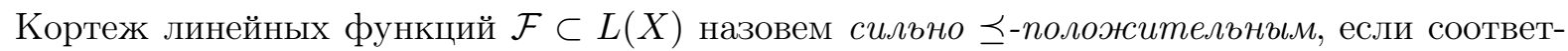
ствующая ему ступенчато-линейная функция $w_{\mathcal{F}}$ является сильно $\preceq$-положительной. 
Следствие. Пусть выпуклая задача векторной оптимизации $(Q, \preceq)$ такова, что $\operatorname{codim} P_{\sim}=m<\infty$, где $P_{\sim}:=\{x \in X \mid x \sim 0\}$. Тогда, для того чтобы $x^{0} \in \operatorname{Min}(Q, \preceq)$,

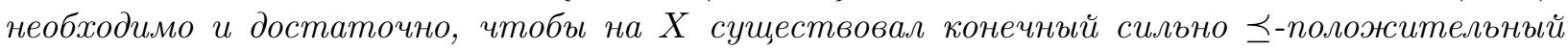
кортежс линейных функиий $\mathcal{F}:=\left\{l_{1}, l_{2}, \ldots, l_{k}\right\}, 1 \leq k \leq m$, такой, что

$$
l_{i}\left(x^{0}\right)=\min _{x \in Q_{i}} l_{i}(x), \quad i=1,2, \ldots, k,
$$

где $Q_{1}=Q, Q_{i+1}:=\left\{z \in X \mid l_{i}(z)=\min _{x \in Q_{i}} l_{i}(x)\right\}, \quad i=1,2, \ldots, k-1$.

Д о к а з а т е л ь с т в о. В силу теоремы $6 x^{0} \in \operatorname{Min}(Q, \preceq)$ тогда и только тогда, когда существует сильно $\preceq$-положительная ступенчато-линейная функция $w: X \rightarrow \mathbb{R}$, удовлетворяющая условию (4.2). Из условия сильной $\preceq$-положительности функции $w$ следует, что $w(x)=0$ для всех $x \in P_{\sim}$, а поскольку $\operatorname{codim} P_{\sim}=m<\infty$, то из условий (б) и (в) из определения кортежа аффинных (линейных) функций заключаем, что число элементов кортежа линейных функций, соответствующего $w$, не превосходит $m=\operatorname{codim} P_{\sim}$.

Итак, пусть $\mathcal{F}:=\left\{l_{1}, l_{2}, \ldots, l_{k}\right\}, 1 \leq k \leq \operatorname{codim} P_{\sim}-$ кортеж линейных функций, который соответствует ступенчато-линейной функции $w: X \rightarrow \mathbb{R}$. Так как функция $w$ сильно $\preceq$-положительна, то кортеж $\mathcal{F}$ также сильно $\preceq$-положителен. Используя представление функции $w: X \rightarrow \mathbb{R}$ в виде (1.2), можно переписать условие (4.2) следующим образом:

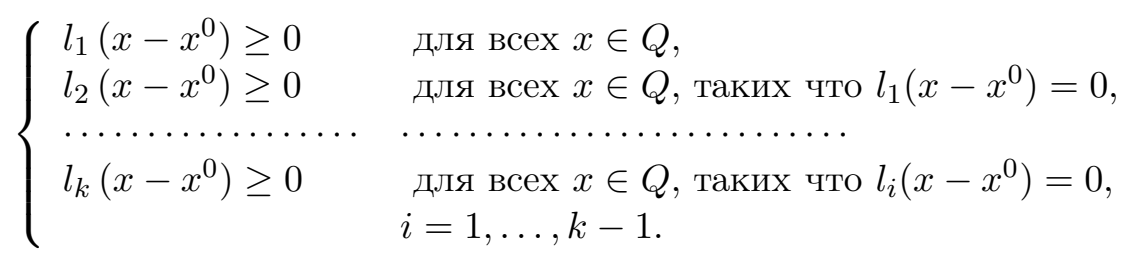

Нетрудно видеть, что совокупность этих условий равносильна (4.7).

\section{5. Критерий оптимальности решений в (нерегулярных по Слейтеру) задачах выпуклого программирования}

Пусть $X$ - вещественное векторное пространство, $f_{i}: X \rightarrow \mathbb{R}, i=0,1, \ldots, m,-$ вещественнозначные выпуклые функции, $Q-$ выпуклое множество из $X$.

Задача выпуклого программирования или, кратко, задача (ВП), состоит в минимизации функции $f_{0}: X \rightarrow \mathbb{R}$ на множестве $\Omega:=\left\{x \in Q \mid f_{i}(x) \leq 0, i=1, \ldots, m\right\}$.

Таким образом, задача (ВП), является задачей оптимизации, множество допустимых решений которой есть определенное выше множество $\Omega$, а отношение предпочтения задается выпуклой функцией полезности $f_{0}: X \rightarrow \mathbb{R}$ (в классической теории оптимизации $f_{0}$ называют также целевой функцией).

Допустимое решение $x^{0} \in \Omega$ называется оптимальным в задаче (ВП), если

$$
f_{0}\left(x^{0}\right) \leq f_{0}(x) \text { для всех } x \in \Omega .
$$

Введем некоторые обозначения. Пусть $I:=\{1,2, \ldots, m\}$. Для каждого $x \in \Omega$ определим множество $I_{a}(x):=\left\{i \in I \mid f_{i}(x)=0\right\}$, называемое множеством индексов, соответствующих ограничениям, активным в точке $x$, или просто множеством индексов активных ограничений в точке $x$. Кроме того, определим для каждого $x \in \Omega$ векторное подпространство

$$
E(x):=\left\{y \in \mathbb{R}^{1+m} \mid y_{0}=0, y_{i}=0, i \in I_{a}(x)\right\} .
$$

Очевидно, что $\operatorname{codim} E(x)=\left|I_{a}(x)\right|+1$, где $\left|I_{a}(x)\right|$ - число элементов множества $I_{a}(x)$.

Введем также отображение $F: X \rightarrow \mathbb{R}^{1+m}$, определив его условием $F: x \rightarrow F(x):=$ $\left(f_{0}(x), f_{1}(x), \ldots, f_{m}(x)\right)$. 
Предложение 7. Допустимое решение $x^{0} \in \Omega$ является оптимальным в задаче (ВП) тогда и только тогда, когда вектор $F\left(x^{0}\right)$ является ${ }_{x^{0}}$-минимальным в множестве $F(\Omega)=$ $\left\{y \in \mathbb{R}^{1+m} \mid y=F(x), x \in \Omega\right\}$, где $\preceq_{x^{0}}-$ согласованное отношение предпорядка на $\mathbb{R}^{1+m}$, конус положительных векторов которого есть

$$
P_{\preceq}\left(x^{0}\right):=\left\{y \in \mathbb{R}^{1+m} \mid y_{0}>0, y_{i} \geq 0, i \in I_{a}\left(x^{0}\right)\right\} \cup E\left(x^{0}\right) .
$$

Д о к а з а т е л ь с т в о. Необходимость. Пусть $x^{0} \in \Omega$ - оптимальное решение задачи (ВП). Предположим вопреки утверждению предложения, что существует вектор $\bar{x} \in \Omega$ такой, что $F(\bar{x}) \prec_{x^{0}} F\left(x^{0}\right)$. Исходя из определений отношения $\preceq_{x^{0}}$ и множества $I_{a}\left(x^{0}\right)$, получаем, что

$$
f_{0}(\bar{x})<f_{0}\left(x^{0}\right), \quad f_{i}(\bar{x}) \leq 0, \quad i \in I_{a}\left(x^{0}\right) .
$$

Рассмотрим вектор $x_{\alpha}=x^{0}+\alpha\left(\bar{x}-x^{0}\right)$. В силу выпуклости множества $Q$ и функций $f_{i}: X \rightarrow$ $\mathbb{R}, i=0,1, \ldots, m$, имеем $x_{\alpha} \in Q$ и $f_{i}\left(x_{\alpha}\right)=f_{i}\left(\alpha \bar{x}+(1-\alpha) x^{0}\right) \leq \alpha f_{i}(\bar{x})+(1-\alpha) f_{i}\left(x^{0}\right)=$ $f_{i}\left(x^{0}\right)+\alpha\left(f_{i}(\bar{x})-f_{i}\left(x^{0}\right)\right)$ для всех $\alpha \in[0,1]$ и всех $i=0, \ldots, m$. Учитывая неравенства $(5.1)$, из предыдущих неравенств получаем

$$
f_{0}\left(x_{\alpha}\right)<f_{0}\left(x^{0}\right), \quad f_{i}\left(x_{\alpha}\right) \leq 0, \quad i \in I_{a}\left(x^{0}\right) \text { для всех } \alpha \in(0,1] .
$$

Кроме того, так как $f_{i}\left(x^{0}\right)<0$ для всех $i \in I \backslash I_{a}\left(x^{0}\right)$, то существует число $\alpha_{0} \in(0,1]$ такое, что $f_{i}\left(x_{\alpha}\right) \leq f_{i}\left(x^{0}\right)+\alpha\left(f_{i}(\bar{x})-f_{i}\left(x^{0}\right)\right) \leq 0$ для всех $i \in I \backslash I_{a}\left(x^{0}\right)$ и всех $\alpha \in\left[0, \alpha_{0}\right)$. Объединив последние неравенства с (5.2), имеем

$$
f_{0}\left(x_{\alpha}\right)<f_{0}\left(x^{0}\right), \quad f_{i}\left(x_{\alpha}\right) \leq 0, \quad i=1,2, \ldots, m, \quad x_{\alpha} \in Q \text { для всех } \alpha \in\left(0, \alpha_{0}\right) .
$$

Это, однако, противоречит оптимальности $x^{0}$ в задаче (ВП). Необходимость доказана.

Достаточность. Условие $\preceq x^{0-м и н и м а л ь н о с т и ~ в е к т о р а ~} F\left(x^{0}\right)$ в $F(\Omega)$ эквивалентно несовместности относительно $x$ системы неравенств

$$
f_{0}(x)<f_{0}\left(x^{0}\right), \quad f_{i}(x) \leq f_{i}\left(x^{0}\right)=0, \quad i \in I_{a}\left(x^{0}\right), \quad x \in Q .
$$

Тем более несовместна относительно $x$ система неравенств

$$
f_{0}(x)<f_{0}\left(x^{0}\right), \quad f_{i}(x) \leq 0, \quad i \in I, \quad x \in Q,
$$

что эквивалентно условию

$$
f_{0}(x) \geq f_{0}\left(x^{0}\right) \text { для всех } x \in \Omega .
$$

Следовательно, $x^{0}$ - оптимальное решение (ВП).

Теорема 7. Допустимое решение $x^{0} \in \Omega$ является оптимальным решением задачи (ВП) тогда и только тогда, когда существуют вектор $\lambda=\left(\lambda_{1}, \lambda_{2}, \ldots, \lambda_{m}\right) \in \mathbb{R}^{m}$ и упорлдоченное разбиение $\left\{I_{1}, I_{2}, \ldots, I_{k}\right\}, 1 \leq k \leq\left|I_{a}\left(x^{0}\right)\right|+1$ множества индексов $I=\{1,2, \ldots, m\}$, удовлетворяющие

а) условию неотрицательности

$$
\lambda_{1} \geq 0, \quad \lambda_{2} \geq 0, \quad \ldots, \quad \lambda_{m} \geq 0
$$

б) условию дополняющей нежесткости

$$
\lambda_{i} f_{i}\left(x^{0}\right)=0, \quad i=1,2, \ldots, m
$$

в) условиям минимума

$$
\begin{aligned}
& \sum_{i \in I_{s}} \lambda_{i} f_{i}\left(x^{0}\right)=\min _{x \in Q_{s}} \sum_{i \in I_{s}} \lambda_{i} f_{i}(x), \quad s=1,2, \ldots, k-1, \\
& f_{0}\left(x^{0}\right)+\sum_{i \in I_{k}} \lambda_{i} f_{i}\left(x^{0}\right)=\min _{x \in Q_{k}}\left(f_{0}(x)+\sum_{i \in I_{k}} \lambda_{i} f_{i}(x)\right),
\end{aligned}
$$

где $Q_{1}=Q, Q_{s+1}=\left\{x \in Q_{s} \mid \sum_{i \in I_{s}} \lambda_{i} f_{i}(x)=\min _{z \in Q_{s}} \sum_{i \in I_{s}} \lambda_{i} f_{i}(z)\right\}, s=1,2, \ldots, k-1$. 
Д о к а з а т е л ь с т в о. В силу предложения 7 допустимое решение $x^{0} \in \Omega$ оптимально в задаче (ВП) тогда и только тогда, когда вектор $F\left(x^{0}\right) \preceq x^{0}$-минимальный в множестве $F(\Omega):=\left\{y \in \mathbb{R}^{1+m} \mid y=F(x), x \in \Omega\right\}$. Используя выпуклость множества $Q$ и функций $f_{i}: X \rightarrow \mathbb{R}, i=0,1, \ldots, m$, нетрудно убедиться в том, что множество $\widehat{F}(Q):=\left\{z \in \mathbb{R}^{1+m} \mid\right.$ $\exists y \in F(Q)$ такой, что $\left.y \preceq \preceq_{x^{0}} z\right\}$ также выпукло. Следовательно, задача векторной оптимизации $\left(F(Q), \preceq_{x^{0}}\right)$ выпукла и к ней применим критерий минимальности, доказанный в теореме 1 .

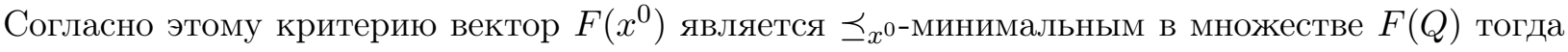
и только тогда, когда существует сильно $\preceq_{x^{0}}$-положительная ступенчато-линейная функция $w: \mathbb{R}^{1+m} \rightarrow \mathbb{R}$ такая, что

$$
w\left(F(x)-F\left(x^{0}\right)\right) \geq 0 \text { для всех } x \in Q .
$$

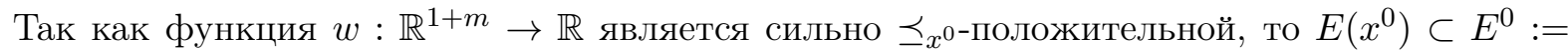
$\{x \in X \mid w(x)=0\}$ и, следовательно, $\operatorname{codim} E^{0} \leq \operatorname{codim} E\left(x^{0}\right)=\left|I\left(x^{0}\right)\right|+1$. Из этого замечания и предложения 1 следует, что в $\mathbb{R}^{1+m}$ существует ортогональный сильно $\preceq x^{0-п о л о ж и т е л ь н ы и ̆ ~}$ кортеж векторов $\Lambda=\left\{\lambda^{(1)}, \lambda^{(2)}, \ldots, \lambda^{(k)}\right\}\left(1 \leq k \leq\left|I_{a}(x)\right|+1\right)$ такой, что ступенчато-линейная функция $w: \mathbb{R}^{1+m} \rightarrow \mathbb{R}$ представима с его помощью в виде (1.3). Используя это представление, нетрудно показать, что (5.5) эквивалентно условиям

$$
\left(\lambda^{(i)}, F\left(x^{0}\right)\right)=\min _{x \in Q_{i}}\left(\lambda^{(i)}, F(x)\right), \quad i=1,2, \ldots, k ;
$$

где $Q_{1}=Q, Q_{i+1}=\left\{x \in Q_{i} \mid\left(\lambda^{(i)}, F(x)\right)=\min _{z \in Q_{i}}\left(\lambda^{(i)}, F(z)\right)\right\}, i=1,2, \ldots, k-1$.

С учетом конкретного вида конуса $P_{\preceq\left(x^{0}\right)}$ и ортогональности кортежа $\Lambda=\left\{\lambda^{(1)}, \lambda^{(2)}, \ldots\right.$, $\left.\lambda^{(k)}\right\}$ непосредственно проверяется, что условие сильной $\preceq x^{0}$-положительности кортежа $\Lambda$ эквивалентно условиям

1) $\lambda_{0}^{(1)}=\lambda_{0}^{(2)}=\ldots=\lambda_{0}^{(k-1)}=0, \lambda_{0}^{(k)}>0$

2) существует разбиение $\left\{J_{1}, J_{2}, \ldots, J_{k}\right\}$ множества индексов $I_{a}\left(x^{0}\right)$ такое, что

$$
\lambda_{i}^{(s)} \geq 0, \quad i \in J_{s}, \quad \lambda_{i}^{(s)}=0, \quad i \in I \backslash J_{s} ; \quad s=1,2, \ldots, k .
$$

Заметим, что число подмножеств в разбиении $\left\{J_{1}, J_{2}, \ldots, J_{k}\right\}$ множества индексов $I_{a}\left(x^{0}\right)$ равно рангу ступенчато-линейной функции $w$, удовлетворяющей условию (5.5).

Нетрудно видеть, что семейство $\left\{I_{1}, I_{2}, \ldots, I_{k}\right\}$, где $I_{s}=J_{s}$ для $s=1,2, \ldots, k-1$ и $I_{k}=J_{k} \cup\left(I \backslash I_{a}\left(x^{0}\right)\right)$, является разбиением множества индексов $I=\{1,2, \ldots, m\}$. Используя векторы кортежа $\Lambda=\left\{\lambda^{(1)}, \lambda^{(2)}, \ldots, \lambda^{(k)}\right\}$ образуем вектор $\lambda=\left\{\lambda_{1}, \lambda_{2}, \ldots, \lambda_{m}\right\} \in \mathbb{R}^{m}$ такой, что $\lambda_{i}=\lambda_{i}^{(s)}$ для $i \in I_{s}, s=1,2, \ldots, k-1$, и $\lambda_{i}=\lambda_{i}^{(k)} / \lambda_{0}^{(k)}$ для $i \in I_{k}$. Кортеж $\Lambda$ удовлетворяет указанным выше свойствам 1) и 2); отсюда следует, что вектор $\lambda=\left\{\lambda_{1}, \lambda_{2}, \ldots, \lambda_{m}\right\}$ удовлетворяет условиям а) и б) теоремы, а из условий (5.6) выводим выполнение условий минимума (5.3) и (5.4). Необходимость доказана.

Достаточность. Из условий а), б) и (5.3) следует, что множество допустимых точек $\Omega=$ $\left\{x \in Q \mid f_{i}(x) \leq 0, i=1, \ldots, m\right\}$ принадлежит каждому $Q_{s}, s=1,2, \ldots, k$, и, в частности, $\Omega$ принадлежит $Q_{k}$. Учитывая это, из условия (5.4) и условий а), б) получаем, что

$$
f_{0}\left(x^{0}\right) \leq f_{0}(x)+\sum_{i \in I_{k}} \lambda_{i} f_{i}(x) \leq f_{0}(x)
$$

для всех $x \in \Omega$, а это и означает оптимальность $x^{0} \in \Omega$ в задаче (ВП).

3 а м е ч а н и е 2. Если для задачи (ВП) выполняется условие регулярности Слейтера, что означает существование точки $\hat{x} \in \mathbb{R}^{n}$ такой, что $f_{i}(\hat{x})<0$ для всех $i=1,2, \ldots, m$, то в доказательстве необходимой части теоремы 7 можно показать, что ранг ступенчато-линейной функции $w: \mathbb{R}^{1+m} \rightarrow \mathbb{R}$, удовлетворяющей условию (5.5), равен единице, т.е. фактически 
$w(y)=l(y), y \in \mathbb{R}^{1+m}$, где $l-$ линейная функция на $\mathbb{R}^{1+m}$. Поскольку число $k$ подмножеств в разбиении множества индексов $I$ на подмножества $J_{1}, \ldots, J_{k}$ равно рангу функции $w$, то при выполнении условий регулярности Слейтера разбиение в теореме 7 совпадает с самим $I$, при этом условия а)-в) совпадают с критерием Куна - Таккера. Таким образом, доказанный в теореме 7 критерий оптимальности решений задачи (ВП) является распространением критерия Куна - Таккера на нерегулярные по Слейтеру задачи.

\section{Заключение}

В работе представлены аналитические критерии непересекаемости выпуклых подмножеств вещественного векторного пространста, основанные на отделимости выпуклых множеств ступенчато-линейными и ступенчато-аффинными функциями. Данные критерии являются, по существу, аналитическими версиями критерия Какутани - Тьюки об отделимости выпуклых множеств полупространствами. Интерес к данным критериям обусловлен их приложениями к нерегулярным выпуклым задачам оптимизации, исследования которых невозможны при помощи классических теорем об отделимости выпуклых множеств гиперплоскостями; некоторые из таких приложений представлены в работе.

Существует ряд открытых вопросов, связанных с отделимостью выпуклых множеств полупространствами или, эквивалентно, отделимостью ступенчато-аффинными функциями. В частности, неясно, при каких дополнительных условиях непересекающиеся выпуклые множества отделимы именно дедекиндовым (недедекиндовым) полупространством (эквивалентно, именно регулярной (сингулярной) ступенчато-аффинной функцией). Неизвестно также, как определить по непересекающимся выпуклым множествам отделяющее их полупространство наименьшего ранга и всегда ли существует такое полупространство.

\section{СПИСОК ЛИТЕРАТУРЫ}

1. Райков Д.Ф. Векторные пространства. М.: Физматгиз, 1962. 212 с.

2. Klee V. Separation and support properties of convex sets. A survey // Control Theory and the Calculus of Variations /ed. A.V. Balakrishnan. N Y: Acad. Press, 1969. P. 235-303.

3. Kakutani S. Ein Beweis des Satzen von M. Eidelheit über konvexe Mengen // Proc. Imp. Acad. Tokio. 1938. Vol. 14. P. 93-94. doi: 10.3792/pia/1195579980.

4. Tukey J.W. Some notes on the separation of convex sets // Portugaliae Math. 1942. Vol. 3, no. 2. P. $95-102$.

5. Хилле Э., Филлипс Р. Функциональный анализ и полугруппы. М.: ИЛ, 1962. 830 с.

6. Martinez-Legaz J.-E., Singer I. The structure of hemispaces in $\mathbb{R}^{n} / /$ Linear Algebra Appl. 1988. Vol. 110. P. 117-179. doi: 10.1016/0024-3795(83)90135-0 .

7. Гороховик В.В. Минимальность и квазиминимальность в упорядоченных векторных пространствах // Докл. АН БССР. 1981. Т. 25, № 8. С. 685-688.

8. Гороховик В.В. Выпуклые и негладкие задачи векторной оптимизации. Минск: Наука и Техника, 1990. $240 \mathrm{c}$.

9. Гороховик В.В., Семенкова Е.А. Ступенчато-линейные функции в конечномерных векторных пространствах. Определение, свойства и их связь с полупространствами // Докл. АН Беларуси. 1997. T. 41, № 5. C. 10-14.

10. Гороховик В.В., Семенкова Е.А. Классификация полупространств по типам в бесконечномерных векторных пространствах // Мат. заметки. 1998. Т. 64, вып. 2. С. 191-198.

11. Гороховик В.В., Шинкевич Е.А. Теоремы об отделимости выпуклых множеств ступенчато-линейными функциями и их приложения к выпуклым задачам оптимизации // Нелинейный анализ и приложения / Национальная Академия наук Беларуси. Труды Института математики. 1998. T. 1. C. $58-85$. 
12. Гороховик В.В., Шинкевич Е.А. Аналитическое представление бесконечномерных полупространств ступенчато-аффинными функциями // Нелинейный анализ и смежные вопросы / Национальная Академия наук Беларуси. Труды Института математики. 1999. Т. 2. С. 63-72.

13. Gorokhovik V.V., Shinkevich E.A. Geometric structure and classification of infinite--dimensional halfspaces // Algebraic Analysis and Related Topics. Banach Center Publications. Vol. 53. Warsaw: Institute of Mathematics PAN, 2000. P. 121-138.

14. Martinez-Legaz J.-E., Vicente-Perez J. Lexicographical representation of convex sets // J. Convex Analysis. 2012. Vol. 19, no. 2. P. 485-496.

15. Küçük M., Soyertem M., Küçük Ya. On constructing total orders and solving vector optimization problems with total orders // J. Global Optim. 2011. Vol. 50, no. 2. P. 235-247. doi: $10.1007 / \mathrm{s} 10898-010-9576-\mathrm{y}$.

16. Küçük M., Soyertem M., Küçük Ya. The generalization of total ordering cones and vectorization to separable Hilbert spaces // J. Math. Anal. Appl. 2012. Vol. 389, no. 2. P. 1344-1351. doi: 10.1016/j.jmaa.2012.01.017.

17. Klee V. The structure of semispaces // Math. Scand. 1956. Vol. 4. P. 54-64. doi: $10.7146 /$ math.scand.a-10455.

18. Klee V. Maximal separation theorems for convex sets // Trans. Amer. Math. Soc. 1968. Vol. 134, no. 1. P. 133-147. doi: 10.1090/S0002-9947-1968-0235457-9.

19. Лейхтвейс К.М. Выпуклые множества. М.: Наука, 1985. 336 с.

20. Семенкова Е.А. Об аналитическом представлении полупространств в конечномерных векторных пространствах // Изв. АН Беларуси. Сер. физ.-мат. наук. 1996. № 2. С. 35-40.

21. Hammer P.C. Maximal convex sets // Duke Math. J. 1955. Vol. 22. P. 103-106. doi: 10.1215/S0012-7094-55-02209-2 .

22. Lassak M. Convex half-spaces // Fund. Math. 1984. Vol. 120, no. 1. P. 7-13. doi: 10.4064/fm-120-1-7-13 .

23. Lassak M.A., Prószyński A. Translate-inclusive sets, orderings and convex halfspaces // Bull. Polish Acad. Sci. Math. 1986. Vol. 34, no. 3-4. P. 195-201.

24. Martinez-Legaz J.-E., Singer I. Lexicographical separation in $\mathbb{R}^{n} / /$ Linear Algebra Appl. 1987. Vol. 90. P. 147-163. doi: 10.1016/0024-3795(87)90312-0 .

25. Александров П.С. Введение в теорию множеств и общую топологию. М.: Наука, 1977. 368 с.

26. Колмогоров А.Н., Фомин С.В. Элементы теории функций и функционального анализа. М.: Наука, 1981. 544 с.

Гороховик Валентин Викентьевич

Поступила 11.11.2019

После доработки 10.01.2020

Принята к публикации 14.01.2020

д-р физ.-мат. наук, профессор

чл.-корр. НАН Беларуси

зав. отделом

Институт математики НАН Беларуси, г. Минск

e-mail: gorokh@im.bas-net.by

\section{REFERENCES}

1. Raikov D.A. Vector Spaces. Groningen: Noordhoff, 1965, 190 p. Original Russian text published in Raikov D.A. Vektornye prostranstva, Moscow: Fizmatgiz Publ., 1962, 212 p.

2. Klee V. Separation and support properties of convex sets. A survey. In: Control Theory and the Calculus of Variations, A.V. Balakrishnan (ed), N Y: Acad. Press, 1969, pp. 235-303. ISBN: 0120769530.

3. Kakutani S. Ein Beweis des Satzen von M. Eidelheit über konvexe Mengen. Proc. Imp. Acad. Tokio, 1938, vol. 14, pp. 93-94. doi: 10.3792/pia/1195579980.

4. Tukey J.W. Some notes on the separation of convex sets. Portugaliae Math., 1942, vol. 3, no. 2, pp. 95102. 
5. Hille E., Phillips R.S. Functional analysis and semi-groups. Coll. Publ., vol. 31, Providence: AMS, 1957, 810 p. ISBN: 0821810316. Translated to Russian under the title Funktsional'nyi analiz $i$ polugruppy. Moscow: IL Publ., 1962, 830 p.

6. Martinez-Legaz J.-E., Singer I. The structure of hemispaces in $\mathbb{R}^{n}$. Linear Algebra Appl., 1988, vol. 110, pp. 117-179. doi: 10.1016/0024-3795(83)90135-0 .

7. Gorokhovik V.V. Minimality and quasiminimality in ordered vector spaces. Dokl. Akad. Nauk BSSR, 1981, vol. 25, no. 8, pp. 685-688 (in Russian).

8. Gorokhovik V.V. Vypuklye i negladkie zadachi vektornoi optimizatsii [Convex and nonsmooth problems of vector optimization]. Minsk: Navuka i Tekhnika Publ., 1990, 240 p. ISBN: 5-4343-00519-5.

9. Gorokhovik V.V., Semenkova E.A. Step-linear functions in finite-dimensional vector spaces. Definition, properties and their relation to half-spaces. Dokl. Akad. Nauk Belarusi, 1997, vol. 41, no. 5, pp. 10-14 (in Russian) .

10. Gorokhovik V.V., Semenkova E.A. Classification of semispaces according to their types in infinitedimensional vector spaces. Math. Notes, 1998, vol. 64, no. 2, pp. 164-169. doi: 10.1007/BF02310300.

11. Gorokhovik V.V., Shinkevich E.A. Theorems on the separation of convex sets by step-linear functions and their applications to convex optimization problems. In: Nonlinear analysis and applications, Tr. Inst. Mat. Nats. Akad. Nauk Belarusi, vol. 1. Minsk: Natl. Akad. Nauk Belarusi, 1998, pp. 58-85 (in Russian) .

12. Gorokhovik V.V., Shinkevich E.A. Analytic representation of infinite-dimensional half-spaces by stepaffine functions. In: Nonlinear analysis and related problems, Tr. Inst. Mat. Nats. Akad. Nauk Belarusi, vol. 2. , Minsk: Natl. Akad. Nauk Belarusi, 1999, pp. 63-72 (in Russian).

13. Gorokhovik V.V., Shinkevich E.A. Geometric structure and classification of infinite-dimensional halfspaces. In: Algebraic Analysis and Related Topics. Banach Center Publications, vol. 53. Warsaw: Institute of Mathematics PAN, 2000, pp. 121-138.

14. Martinez-Legaz J.-E., Vicente-Perez J. Lexicographical representation of convex sets. J. Convex Analysis, 2012, vol. 19, no. 2, pp. 485-496.

15. Küçük M., Soyertem M., Küçük Ya. On constructing total orders and solving vector optimization problems with total orders. J. Global Optim., 2011, vol. 50, no. 2, pp. 235-247. doi: 10.1007/s10898-010-9576-y .

16. Küçük M., Soyertem M., Küçük Ya. The generalization of total ordering cones and vectorization to separable Hilbert spaces. J. Math. Anal. Appl., 2012, vol. 389, no. 2, pp. 1344-1351. doi: 10.1016/j.jmaa.2012.01.017.

17. Klee V. The structure of semispaces. Math. Scand., 1956, vol. 4, pp. 54-64. doi: 10.7146/math.scand.a-10455.

18. Klee V. Maximal separation theorems for convex sets. Trans. Amer. Math. Soc., 1968, vol. 134, no. 1, pp. 133-147. doi: 10.1090/S0002-9947-1968-0235457-9 .

19. Leichtweiss K. Konvexe Mengen. Berlin: Springer, 1980, 330 p. ISBN: 978-3-540-09071-7. Translated to Russian under the title Vypuklye mnozhestva. Moscow: Nauka Publ., 1985, 335 p.

20. Semenkova E.A. On analytical representation of half-spaces in finite-dimensional vector spaces. Izv. Akad. Nauk Belarusi, Ser. Fiz.-Mat. Nauk, 1996, no. 2, pp. 35-40 (in Russian) .

21. Hammer P.C. Maximal convex sets. Duke Math. J., 1955, vol. 22, no. 1, pp. 103-106. doi: 10.1215/S0012-7094-55-02209-2 .

22. Lassak M. Convex half-spaces. Fund. Math., 1984, vol. 120, no. 1, pp. 7-13. doi: 10.4064/fm-120-1-7-13 .

23. Lassak M.A., Prószyński A. Translate-inclusive sets, orderings and convex halfspaces. Bull. Polish Acad. Sci. Math., 1986, vol. 34, no. 3-4, pp. 195-201.

24. Martinez-Legaz J.-E., Singer I. Lexicographical separation in $\mathbb{R}^{n}$. Linear Algebra Appl., 1987, vol. 90, pp. 147-163. doi: 10.1016/0024-3795(87)90312-0 .

25. Alexandroff P.S. Einführung in die Mengenlehre und in die allgemeine Topologie. (Introduction to set theory and to general topology). Berlin: VEB Deutscher Verlag der Wissenschaften, 1984, 336 p. Original Russian text published in Aleksandrov P.S., Vvedenie $v$ teoriyu mnozhestv $i$ obshchuyu topologiyu, Moscow: Nauka Publ., 1977, 368 p. 
26. Kolmogorov A.N., Fomin S.V. Elements of the theory of functions and functional analysis. USA: Martino Fine Books, 2012, 280 p. ISBN: 1614273049. Original Russian text published in Kolmogorov A.N., Fomin S.V., Elementy teorii funktsii i funktsional'nogo analiza, Moscow: Nauka Publ., 1981, 544 p.

Received November 11, 2019

Revised January 10, 2020

Accepted January 14, 2020

Funding Agency: This work was supported by the National Program for Scientific Research of the Republic of Belarus for 2016-2020 "Convergence 2020" (project no. 1.4.01).

Valentin Vikent'evich Gorokhovik, Dr. Phys.-Math. Sci., Corresponding Member of NAS of Belarus, Prof., Institute of Mathematics, The National Academy of Sciences of Belarus, Minsk, 220072 Belarus, e-mail: gorokh@im.bas-net.by .

Cite this article as: V.V. Gorokhovik. Step-affine functions, half-spaces, and separation of convex sets with applications to convex optimization problems, Trudy Instituta Matematiki i Mekhaniki URO RAN, 2020, vol. 26, no. 1, pp. 51-70. 